\title{
Including intraspecific trait variability to avoid distortion of functional diversity and ecological inference: Lessons from natural assemblages
}

\author{
Mark K. L. Wong ${ }^{1,2}$ (D) | Carlos P. Carmona ${ }^{3}$ (i)
}

${ }^{1}$ Department of Zoology, University of Oxford, Oxford, UK

${ }^{2}$ Forest Entomology, Swiss Federal Research Institute WSL, Birmensdorf, Switzerland

${ }^{3}$ Institute of Ecology and Earth Sciences, University of Tartu, Tartu, Estonia

\section{Correspondence}

Mark K. L. Wong

Email: mark.wong@zoo.ox.ac.uk

\section{Funding information}

Estonian Research Council; European Regional Development Fund; National Geographic Society, Grant/Award Number: 60-16; University of Oxford

Handling Editor: Torbjørn Ergon

\section{Abstract}

1. Functional diversity assessments are crucial and increasingly used for understanding ecological processes and managing ecosystems. The functional diversity of a community is assessed by sampling traits at one or more scales (individuals, populations and species) and calculating a summary index of the variation in trait values. However, it remains unclear how the scales at which traits are sampled and the indices used to estimate functional diversity may alter the patterns observed and inferences about ecological processes.

2. For 40 plant and 61 ant communities, we assess functional diversity using six methods-spanning various mean-based and probabilistic methods-that reflect common scenarios where different levels of detail are available in trait data. We test whether including trait variability at different scales (from individuals to species) alters functional diversity values calculated using the volume-based and dissimilarity-based indices, Functional Richness (FRic) and Rao, respectively. We further test whether such effects alter functional diversity patterns observed across communities and their relationships with environmental drivers such as abiotic gradients and occurrences of invasive species.

3. Intraspecific trait variability strongly determined FRic and Rao. Methods using only species' mean trait values to calculate FRic (convex hulls) and Rao (Gowerbased dissimilarity) distorted the patterns observed when intraspecific trait variability was considered. These distortions generated Type I and Type II errors for the effects of environmental factors structuring the plant and ant communities. A high sensitivity of FRic to individuals with extreme trait values was revealed in comparisons of different probabilistic methods including among-individual and among-population trait variability in functional diversity. In contrast, values of and ecological patterns in Rao were consistent among methods including different scales of intraspecific trait variability.

4. Our results show empirically that decisions about where traits are sampled and how trait variability is included in functional diversity can drastically change the patterns observed and conclusions about ecological processes. We recommend 
sampling the traits of multiple individuals per species and capturing their intraspecific trait variability using probabilistic methods. We discuss how intraspecific trait variability can be reasonably estimated and included in functional diversity in the common circumstance where only limited trait data are available.

\section{KEYWORDS}

ant, community assembly, competition, environmental filtering, functional richness, functional trait, limiting similarity, Rao

\section{1 | INTRODUCTION}

Assessments of the diversity of organisms' functional traits'functional diversity'-are important for understanding manifold phenomena ranging from macroevolutionary processes (Díaz et al., 2016; Pigot et al., 2020) to community assembly (McGill et al., 2006) and biodiversity-ecosystem function relationships (Gross et al., 2017). Most functional diversity assessments at and above the community level use just a single value for each trait of each species-the species mean (Mouchet et al., 2010; Villéger et al., 2008). Calculating functional diversity from species-mean trait values lightens demands on trait measurement, especially when diverse ecological communities and large spatial scales are involved. In studies where trait data for species vary in origin or structure (e.g. Díaz et al., 2016; Pigot et al., 2020; Weiss \& Ray, 2020), the species-mean trait value can also be used directly to achieve uniform representation across species, facilitating interspecific comparisons.

The species-mean trait value, however, overlooks trait variability among conspecific individuals, which may be extensive due to effects from local adaptation, phenotypic plasticity, developmental conditions and ontogeny (Des Roches et al., 2018). This intraspecific trait variability can determine species' ecological interactions (Ames et al., 2015; Carmona et al., 2019; Des Roches et al., 2018), and contribute substantially to community functional diversity (as shown by Albert et al., 2011; Messier et al., 2010; Siefert et al., 2015). Assessments failing to account for intraspecific trait variability may therefore misestimate the levels of functional diversity in reality. However, the extent to which these effects alter the observed patterns of functional diversity across communities and inferences about ecological processes are less explored in empirical systems.

Intraspecific trait variability mainly occurs at three hierarchical scales (Albert et al., 2011), each varying in its contribution to functional diversity and relevance to different community processes. At the broadest scale is trait variability among separate local populations. This generally increases as species are distributed across heterogeneous environments. Thus, processes such as environmental filtering may be better detected in functional diversity assessments incorporating population-level trait variability than those surveying species-level trait variability only (Gross et al., 2013). At a finer scale, trait variability among individuals within the same populations affects communities through biotic interactions. Simulation-based, field and experimental studies on plant communities show that accounting for such individual-level trait variability can improve the detection of reduced niche overlap (de Bello et al., 2013; Mason et al., 2011) and the prediction of coexistence outcomes between competing species (Carmona, de Bello, Azcárate, et al., 2019). At the finest scale (and outside the scope of this study), trait variability within an individual may also influence community processes (Westneat et al., 2015). Few empirical studies have compared the effects of individual- to population-level trait variability on functional diversity patterns (but see Messier et al., 2010).

Functional diversity indices summarise the variation in traits at the considered scale. Methods to calculate functional diversity indices can be categorised under two groups. Those in the first group use the trait dissimilarity between species to calculate community functional diversity. Widely used dissimilarity-based indices include Rao's quadratic entropy (Botta-Dukát, 2005), functional dispersion (FDis; Laliberté \& Legendre, 2010), mean pairwise distance (MPD; Weiher et al., 1998) and the FD index of Petchey and Gaston (2002). In these, dissimilarity is often calculated based on Gower's distance, which generally does not incorporate intraspecific trait variability because it uses only the mean trait value for each species (but see Cianciaruso et al., 2009). Gower-based dissimilarity is also affected by the species pool considered, as this determines the range of trait values used to standardise Gower's distances (de Bello et al., 2013). As a less context-dependent alternative, one can compute trait dissimilarity based on the overlap between the trait probability density (TPD) functions of different species (Carmona et al., 2016a; Carmona, de Bello, Mason, et al., 2019). Unlike Gower-based dissimilarity, overlap-based dissimilarity using TPD functions includes intraspecific trait variability.

Methods in the second group use the position of entities (i.e. individuals or species) in a multidimensional trait space to characterise the boundaries of a hypervolume encompassing all trait values observed in the community. The various Functional Richness (FRic) indices calculated using convex hulls (Cornwell et al., 2006), n-dimensional hypervolumes (Blonder et al., 2018; Mammola \& Cardoso, 2020) or TPD functions (Carmona et al., 2016a; Carmona, de Bello, Mason, et al., 2019) are examples of such volume-based indices. Whereas a convex hull is defined by the positions of entities with the most extreme trait values, $n$-dimensional hypervolume and TPD functions estimate a probabilistic hypervolume in which the frequencies of different trait values are accounted 
for (Carmona et al., 2016; Carmona, de Bello, Mason, et al., 2019; Mammola \& Cardoso, 2020).

The different scales at which traits can be sampled, often with limited resources, and the variety of methods for calculating functional diversity indices make functional diversity assessments logistically challenging to implement (van der Plas et al., 2017). Empiricists thus often have to choose, a priori, the scales of trait variability to include (e.g. species-level only, or including population and/or individual levels), the indices used and the methods to calculate them-with the aim of achieving the most unbiased representation of functional diversity patterns. Although dissimilarity- and volume-based functional diversity indices such as Rao and FRic are used widely in empirical studies on functional diversity (Mouchet et al., 2010), there is little information about their sensitivity to different scales of trait variability.

In communities of plants in the Mediterranean region and ants in tropical Asia, we investigate the extent to which excluding different scales of trait variability alter the observed functional diversity patterns and conclusions about the environmental factors driving community structure. We first calculate the FRic and Rao of communities using trait data of the highest available resolution (the greatest number of replicates in the smallest sampling unit, i.e. the plot). These 'HighRes' methods include as many scales of trait variability permitted by the data and use probabilistic distributions (TPD functions) of trait values to calculate indices that should best approximate the functional diversity in reality. The HighRes method for plants includes individual-, population- and species-level trait variability, while that for ants includes individual- and species-level trait variability. We then compare the values of FRic and Rao from HighRes methods to those from other commonly used methods for calculating functional diversity, which include fewer scales of trait variability owing to the lower resolution of the trait data available. Finally, we model the relationships of FRic and Rao against environmental variables, and test whether the patterns captured with HighRes methods are distorted when the other methods are used to calculate functional diversity.

\section{2 | MATERIALS AND METHODS}

\subsection{Community and trait data}

The plant dataset, from Carmona et al. (2015), comprises abundance data for 51 plant species in each of 40 plots distributed along a slope (average inclination: 25\%) in central Spain subjected to a Mediterranean climate. Soils towards the upper part of the slope were shallow and of low nutrient and water availability, whereas soils towards the bottom of the slope were deeper and far more productive. For 10 individuals of each species in each plot, data were collected for two traits, plant height and specific leaf area, producing a trait dataset encompassing 2,540 individuals.

The ant dataset, from Wong et al. (2020), comprises frequencyof-occurrence data for 29 ant species in each of 61 plots in an open tropical grassland in Hong Kong. One species, present in 24 plots, was the Red Imported Fire Ant (Solenopsis invicta), an invasive species known to impact the structure of ant communities (Gotelli \& Arnett, 2000). Data for seven morphological traits (summarised in Table S1) were collected for $\geq 10$ individuals (mean $=11$, $\max .=20$ ) of every species, producing a trait dataset encompassing 319 individuals. This included data from separate sub-castes of polymorphic species (Wong et al., 2020). As far as possible, the selected individuals of each species were chosen to reflect the range of body sizes encountered across all samples. Digital photographs taken at every plot were used to estimate percentage ground cover via colour thresholding techniques in ImageJ (Abramoff, 2004).

\subsection{Dimension reduction for ant traits and data preparation}

We used principal component analysis (PCA) to synthesise the major independent axes of variation in multidimensional trait space captured by the seven traits in the ant dataset (after Martello et al., 2018). The trait measurements (except body size) were first size-corrected by dividing by the measurement for body size (Weber's Length). All traits were then log-transformed and standardised to have a mean of 0 and a standard deviation of 1 . We then performed the PCA using the mean trait values of each species, and retained the first two principal components, which had eigenvalues greater than unity. Next, we projected the trait values for each individual $(N=319)$ in the trait dataset into the two principal components (using the function 'predict' in R); individuals' values for these two new 'traits' were used for all subsequent assessments of ant functional diversity. We used species means instead of individual trait values to perform the PCA because using the latter could bias the analysis if some species had disproportionately large numbers of individuals in the dataset (Martello et al., 2018). Trait data for plants were log-transformed and standardised prior to functional diversity calculations.

\section{3 | Calculating functional diversity indices with different scales of trait variability}

We used six different methods to calculate the FRic and Rao of every community (plot) in both the plant and ant datasets (Table 1). The methods differ generally in the scales of trait variability included in community functional diversity (Table 1), and specifically in the ways by which trait variability is scaled up to community functional diversity (see descriptions of individual methods below). They encompass an array of options available to researchers performing functional diversity analyses, and correspond to different scenarios, across which the required resolution of the trait data varies. The methods are ordered from high resolution (those systematically sampling trait values of individuals within populations and using these values to estimate probabilistic trait distributions of species directly) to low 
TAB LE 1 Methods for assessing functional diversity based on the resolution of trait data. A given method may include up to three hierarchical scales of trait variability: among individuals within the same populations (Individuals), among separate local populations (Populations) and among different species (Species). Methods differ in whether trait variability at each scale has been sampled systematically and included $(\checkmark)$, not sampled systematically but still included $(\sim)$ or not included entirely $(\boldsymbol{X})$. All methods estimate functional diversity by combining species' relative abundances in the community with the distributions of their trait values at the finest available scale. At that scale, the distribution of trait values of each species can be estimated directly (using a kernel density function) as a trait probability density function for that species $\left(T_{P D}\right.$ ) if many conspecific individuals have been measured; if not, the $\operatorname{TPD}_{S}$ can be estimated as a multivariate normal distribution centred in the mean trait value, or the trait distribution can be substituted by the mean trait value entirely. Methods were applied to different taxa. For each taxon, the method using the highest available resolution in the trait data is indicated $\left({ }^{*}\right)$. See main text for full details of individual methods

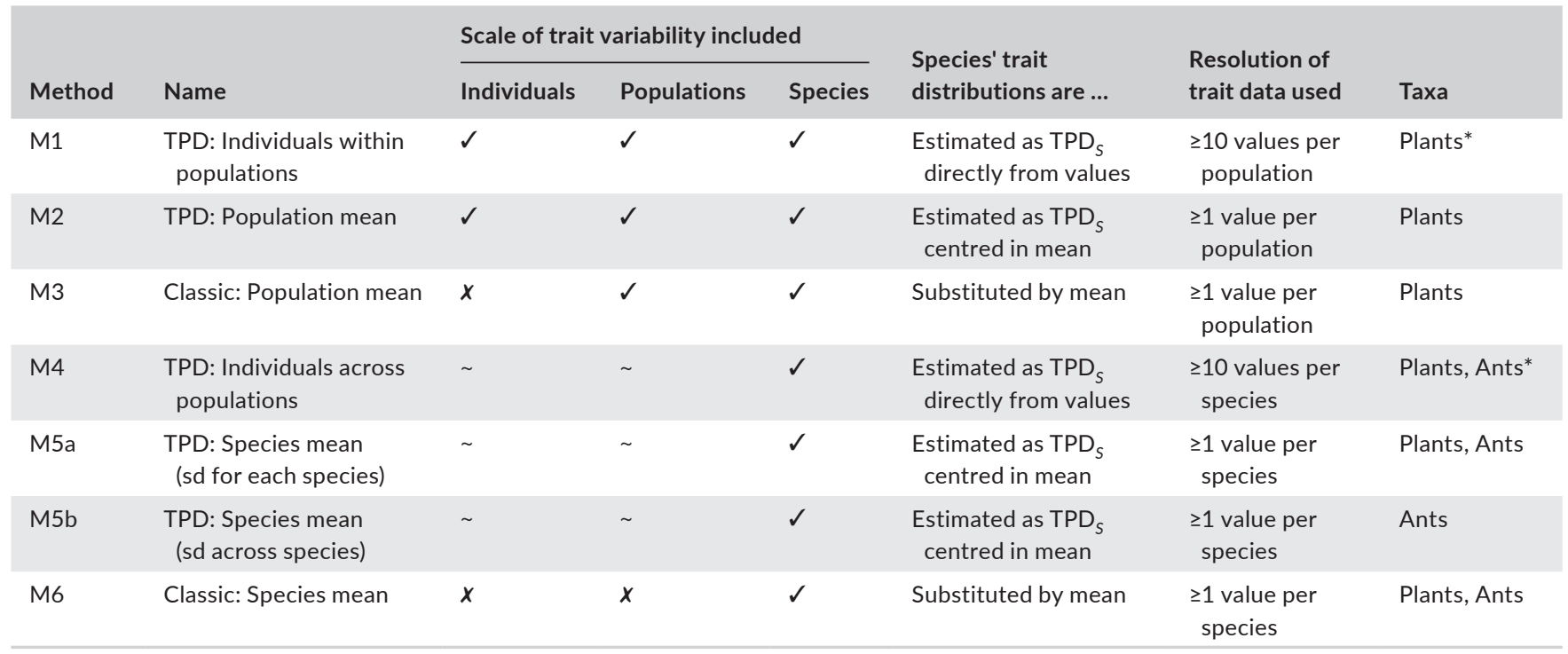

resolution (those using only the mean trait value of each species to calculate functional diversity indices).

\subsection{1 | M1. TPD: Individuals within populations}

The various trait probability density (TPD) methods involve generating a trait probability density function for each species-the $\mathrm{TPD}_{S}-$ which probabilistically summarises the distribution of a given species' trait values at a given scale. The $\mathrm{TPD}_{S}$ functions of different species are weighted using species' relative abundances and aggregated to produce the trait probabilistic density function for the community at every plot-the TPD ${ }_{C}$. Functional diversity indices are then calculated based on properties of the TPD ${ }_{C}$ (for FRic) as well as the relationships between the $\mathrm{TPD}_{S}$ of different species (for Rao).

M1 includes the intraspecific trait variability among individuals in populations as well as among different populations. It can be used in a 'best-case' scenario where the traits of many individuals within each population have been measured systematically such that one $\mathrm{TPD}_{S}$ for each population of a species can be estimated directly using a kernel density function and the trait values acquired from that population (Carmona et al., 2016; Carmona, de Bello, Mason, et al., 2019).

To execute $\mathrm{M} 1$, we first estimated one $\mathrm{TPD}_{S}$ for each population of a species using kernel density functions and the trait values of conspecific individuals measured in each plot (henceforth, we use 'population' in reference to conspecifics within the same plot). In doing so, intraspecific trait variability among individuals in the same population was captured by the $\mathrm{TPD}_{S}$ at a plot while that among populations was captured by the $\mathrm{TPD}_{S}$ across different plots. We then aggregated the $\mathrm{TPD}_{S}$ of the different species in each plot according to their relative abundances to estimate the TPD ${ }_{C}$. We calculated FRic as the volume of the $\mathrm{TPD}_{C}$, and Rao from the dissimilarity among the $\mathrm{TPD}_{S}$ of the species present. A similar approach has been used in Carmona, de Bello, Azcárate, et al. (2019).

\subsection{2 | M2. TPD: Population mean}

This TPD method includes intraspecific trait variability among different populations. With a smaller degree of precision than M1, it also includes the intraspecific trait variability among individuals within the same population by likewise estimating one $\mathrm{TPD}_{S}$ for every population of a species. However, instead of using a kernel density function to estimate the $\mathrm{TPD}_{S}$ directly from the trait values of individuals (as in M1), M2 uses a variance estimation approach to estimate the $\mathrm{TPD}_{S}$ as a multivariate normal distribution centred in the mean trait value in the population (as proposed by Carmona et al., 2016a). It assigns the same among-individual trait variability to all species occurring within the same plot while doing this. This method is relevant to a scenario where at least one individual from each population (plot) is measured, but the sample size is deemed insufficient for using a kernel density function to estimate $\mathrm{TPD}_{S}$ directly from the trait values acquired (while there are no studies examining the minimum 
sample sizes for estimating $\mathrm{TPD}_{S}$ with different trait dimensions, Blonder (2016) recommends using at least a number of observations $m$ such that $\log (m)>$ number of dimensions).

To execute $M 2$, we estimated a $\mathrm{TPD}_{S}$ function for each population using the TPDsMean function from the TPD package (Carmona, de Bello, Mason, et al., 2019). In this case, the resultant $\mathrm{TPD}_{S}$ for each species in each plot was a multivariate normal distribution centred in the mean trait value among its individuals in that plot, with a standard deviation determined by the estimated bandwidth (across all species in the same plot). The bandwidth was estimated by applying the Hpi.diag function from the ks package (Duong, 2015); further details on the significance of bandwidth selection and potential alternatives to estimating bandwidths are discussed in Carmona, de Bello, Mason, et al. (2019). Once the TPD ${ }_{S}$ was estimated, the aggregation of $\mathrm{TPD}_{S}$ to $\mathrm{TPD}_{C}$ at each plot and the calculation of the FRic and Rao of each community were performed as in M1.

\subsection{3 | M3. Classic: Population mean}

The various 'Classic' methods disregard the intraspecific trait variability among individuals in the same populations because they assign the same trait value to all conspecific individuals in each plot. Classic methods calculate FRic using convex hulls and Rao using Gower-based dissimilarities. M3 includes intraspecific trait variability among different populations. It can be used in a scenario where at least one individual from each population (plot) is measured for each species (similar to $\mathrm{M} 2$ ).

To execute M3, we first calculated the mean trait value of each population of each species (as in M2). Next, we used those mean trait values of species in populations to calculate the FRic and Rao of the community at each plot directly (i.e. no TPD functions were calculated). We calculated FRic using the convex hull method implemented in the dbFD function from the FD package (Laliberté et al., 2014), and Rao with the Gower dissimilarity matrix between populations using the 'melodic' R function (de Bello et al., 2016). A similar approach has been used in Carmona et al. (2015) and Gross et al. (2013).

\subsection{4 | M4. TPD: Individuals across populations}

This TPD method includes intraspecific variability among the individuals of each species and treats the trait structure of species and communities in a probabilistic way; however, it does not strictly include the effects of differences between populations on traits. It can be used in a scenario where trait values have not been systematically acquired across the individuals and populations sampled, but sample sizes are nonetheless sufficient for using kernel density functions to directly estimate one $\mathrm{TPD}_{S}$ for each species as a whole (instead of one $\mathrm{TPD}_{S}$ per population per species as in $\mathrm{M} 1$ and $\mathrm{M} 2$ ). For instance, some investigators may arbitrarily measure the traits of many individuals per species, or intentionally measure the traits of the smallest and largest individuals to capture the variability of the species while ignoring the distribution of those individuals across the plots sampled.

To execute M4, we estimated one $\mathrm{TPD}_{S}$ for each species directly using a kernel density function and the trait values of all its individuals in the dataset. At each plot, the aggregation of $T P D_{S}$ to $T P D_{C}$ and calculation of the FRic and Rao of the community were performed as in M1. A similar approach has been used in Traba et al. (2017).

\subsection{5 | M5. TPD: Species mean}

This TPD method includes intraspecific trait variability of a similar structure as that of M4 but forgoes some precision to relax demands on sample size. Like M4, M5 estimates one $T_{P D}$ for each species only. However, it uses a variance estimator to estimate the $\mathrm{TPD}_{S}$ as a multivariate normal distribution centred in the species' mean trait value (similar to M2). M5 is thus most relevant to a scenario where insufficient individuals of each species have been measured to allow for a direct estimation of the $\mathrm{TPD}_{S}$ using a kernel density function.

To execute M5, we first calculated the mean trait value of each species from the trait values of all its individuals in the dataset. We then used the TPDsMean function, as in M2-but here we explored two alternative approaches for assigning the bandwidths (variances). These approaches reflect scenarios researchers encounter frequently.

In the first, M5a. TPD: species mean (sd for each species), the bandwidth used for estimating the $\mathrm{TPD}_{S}$ of each species was the standard deviation of all available trait values of that particular species (after Martello et al., 2018). This approach corresponds to the scenario where multiple trait values are available for each species, but the sample size is insufficient for using a kernel density function to estimate $\mathrm{TPD}_{S}$ directly from those values. Still, this method assigns to each species an amount of intraspecific trait variability that reflects the trait differences between conspecifics observed in reality (Lamanna et al., 2014).

The second approach, M5b. TPD: species (sd across species), was applied to the ant dataset only. This approach can be used in a scenario where only one trait value is available for each species-a common limitation in studies using trait information from the literature or databases. Here, we followed Lamanna et al. (2014) and estimated the $\mathrm{TPD}_{S}$ of every species using a constant bandwidth value: 0.5 times the standard deviation of the trait values of all species in the dataset.

Once the $\mathrm{TPD}_{S}$ of all species was estimated via M5a or M5b, the aggregation of $\mathrm{TPD}_{S}$ to $\mathrm{TPD}_{C}$ at each plot and the calculation of the FRic and Rao of each community were performed as in M1.

\subsection{6 | M6. Classic: Species mean}

This method excludes intraspecific trait variability entirely and includes interspecific trait variability only. It is the most widely used approach in functional diversity assessments $(>1,000$ citations on 
Google Scholar for package FD; Laliberté \& Legendre, 2010), as it only requires a single trait value (e.g. the species mean) for each species and does not involve the estimation of TPD functions.

To execute M6, we first calculated the mean trait value of each species from the trait values of all its individuals in the dataset. We then calculated the FRic and Rao of each community at each plot directly, following the same procedure as in M3.

All methods except M5b were applied to the plant data, whereas M4-M6 were applied to the ant data because trait measurements of the ants were not associated with specific plots. We designated M1 and M4 as the HighRes models for plants and ants, respectively. All functional diversity analyses were performed in R ( $R$ Core Team, 2017); those involving TPD were performed using the TPD package (Carmona, de Bello, Mason, et al., 2019) while those involving convex hull volumes were performed using the FD and BETAPART (Baselga et al., 2018) packages. Further details on the calculation of FRic and Rao using the two broad methodological frameworks presented (TPD and Classic), and the general relationships between TPD and Classic as revealed from simulated data are found in the Supporting Information.

\section{4 | Statistical analyses}

To investigate the relationships among different methods, we analysed the Pearson's correlation between the values of each functional diversity index (i.e. FRic or Rao) calculated by the different methods with the plant and ant data. This allowed us to identify methods which yielded more similar results to the designated HighRes model overall.

We investigated whether different methods captured the same ecological patterns. For the plant data, we investigated the changes in FRic and Rao in response to changes in water availability (percentage water content in soil samples taken from each plot) along the slope. After visually inspecting the shapes of the relationships between the functional diversity indices and water availability, we fitted, for each index and method, a regression using water availability and its quadratic and cubic terms. For the ant data, we examined the changes in FRic and Rao in response to changes in percentage ground cover, and tested whether these patterns varied depending on the presence of the invasive species, S. invicta. A visual inspection of the response of functional diversity indices to percentage ground cover suggested a more linear response than for plants, but with different responses between invasion statuses. Accordingly, for each index and method, we fitted a regression where we considered linear and quadratic terms for percentage ground cover, invasion status (as a binary variable: 'invaded' vs. 'not invaded'), and the interaction between them as predictors.

We then reproduced a model selection process to investigate whether the different methods used led to different ecological conclusions. We used the MuMIN package in R (Barton, 2016) to generate all potential subsets of all models, and ranked them using the Akaike information criterion corrected for small sample sizes (AICC).
We selected the model from the HighRes method with the lowest $\mathrm{AICc}$ value as the one that best reflected the ecological patterns in each dataset. For each of the other candidate methods, we represented the results of the model with the lowest AICC value graphically, and calculated its $\triangle \mathrm{AICc}$ (difference in AICc score) with respect to the selected HighRes model. Other alternatives to model selection are certainly possible, such as using likelihood ratio tests between nested models to retain only statistically significant variables, or model averaging based on Akaike weights (e.g. Carmona, de Bello, Azcárate, et al., 2019). We opted for AICc-based model selection because it is very widely used; for instance, both the 'step' $\mathrm{R}$ function and the default option in the 'dredge' function from the MuMIN package are based on AIC scores. Furthermore, this framework provided a straightforward way to rank models in terms of their performance.

With respect to the results, an $\triangle \mathrm{AICc}$ value of 0 would indicate that the candidate method led to a similar ecological interpretation as the HighRes method. Relatively small $\Delta$ AICc values (e.g. $\Delta \mathrm{AIC}<2$ ) would indicate that, while leading to different ecological interpretations, the HighRes model was not deemed as completely implausible under the candidate method. High $\triangle \mathrm{AICc}$ values (e.g. $\Delta \mathrm{AICc}>2$ ) would imply that the functional diversity values and patterns produced by the candidate method would lead to substantially different ecological interpretations from the results of the HighRes method (Burnham \& Anderson, 2002).

\section{3 | RESULTS}

\subsection{Correlations between functional diversity indices calculated by different methods}

In analyses for plants, the similarities between the HighRes method (M1) and the other methods (M2-M6) in their calculated FRic and Rao did not show a clear trend with the resolution of trait data and the scales of trait variability included (Figure 1). Instead, for both indices, the values calculated with M5a and M4 were most similar to those calculated with $\mathrm{M} 1\left(\rho_{\text {FRic }}=0.82, \rho_{\text {Rao }}=0.87\right.$ for M5a; and $\rho_{\text {FRic }}=0.74, \rho_{\text {Rao }}=0.83$ for M4). All other methods largely failed to obtain FRic values similar to those from M1 $(\rho \leq 0.51)$ but performed better where Rao was concerned ( $\rho \geq 0.73$ ). In analyses for ants, the similarities between the HighRes method (M4) and the other methods (M5a, M5b, M6) in their FRic and Rao generally decreased with decreasing resolution in trait data and as fewer scales of trait variability were included (Figure 2); M5a performed especially well $\left(\rho_{\text {FRic }}=0.97 ; \rho_{\text {Rao }}=0.99\right)$ while M6 performed the worst $\left(\rho_{\text {FRic }}=0.82\right.$; $\rho_{\text {Rao }}=0.66$ ).

\section{2 | FRic responds to the exclusion of specific scales of intraspecific trait variability}

The FRic patterns detected by the best models of the respective HighRes methods for both the plant and ant data were not likewise 
(a) FRic

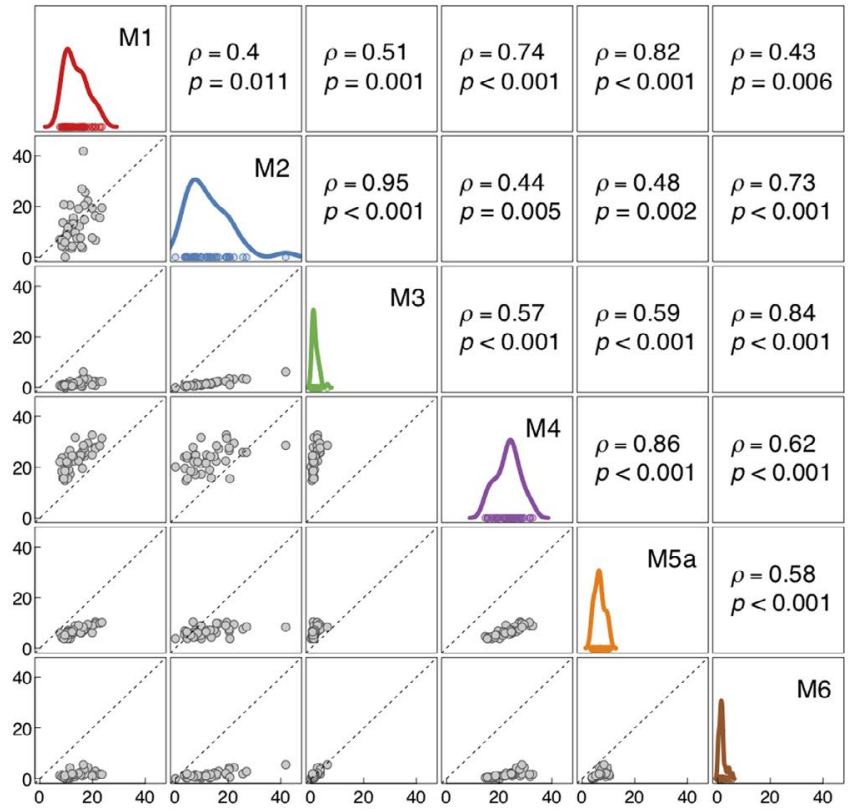

(b) Rao

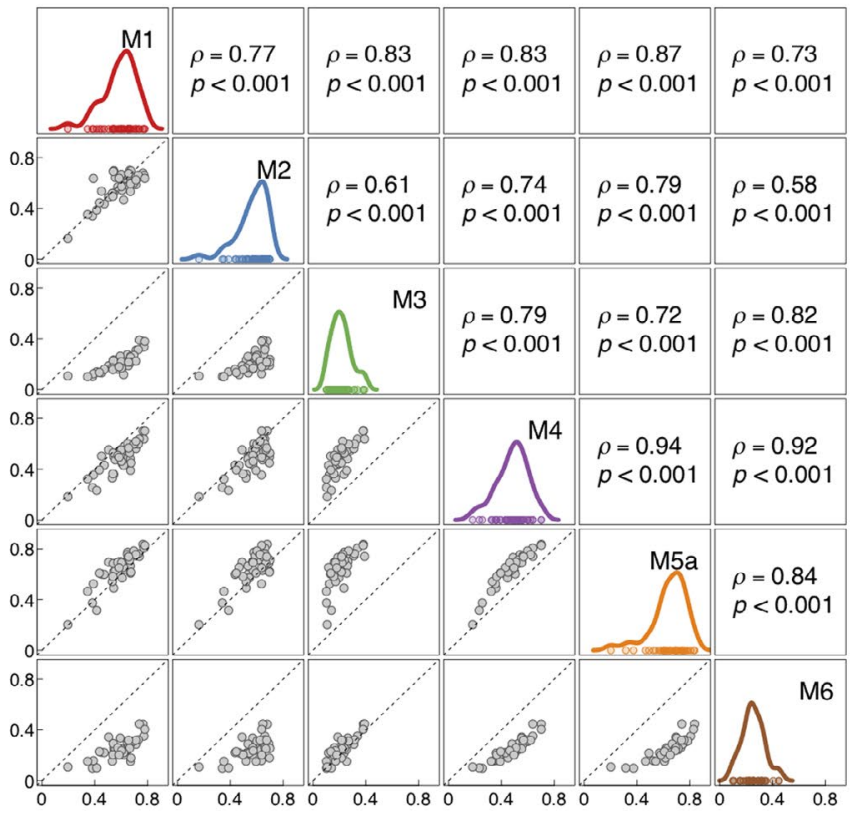

FIGURE 1 For 40 plant communities, plots show the degree of similarity, as measured by Pearson's correlation ( $\rho$ ), in values of Functional Richness (FRic) (a) and Rao (b) calculated by different methods. Each method (M1-M6) includes a different scale (or scales) of trait variability in functional diversity, based on the resolution of the trait data (see Table 1). Plots along the diagonal depict probability density functions showing the distribution of FRic or Rao values calculated by individual methods

\section{(a) FRic}

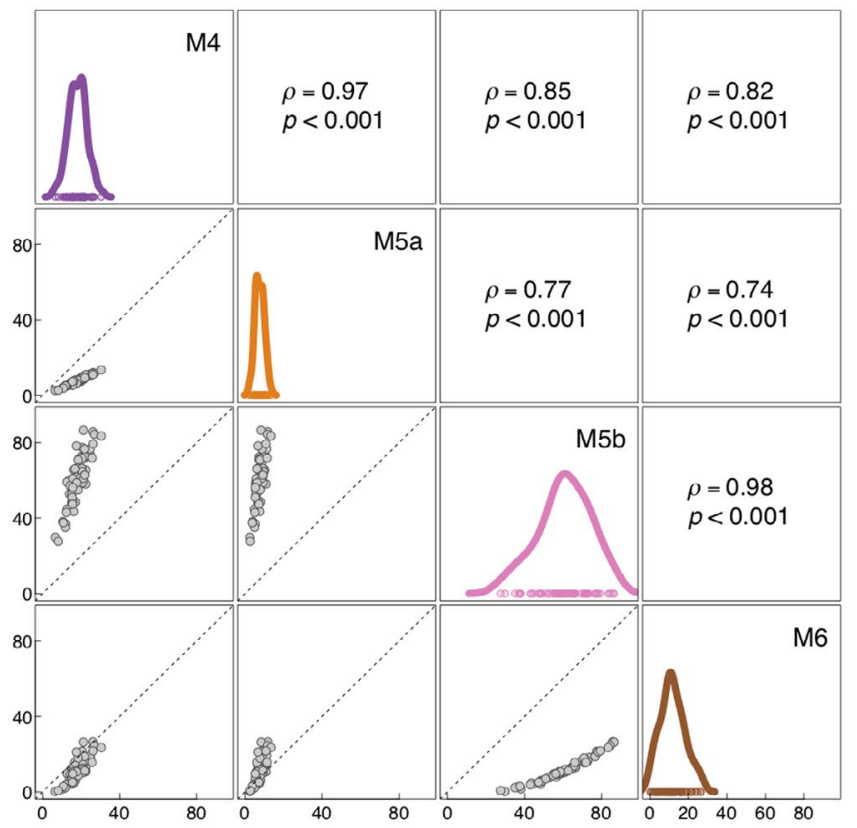

(b) Rao

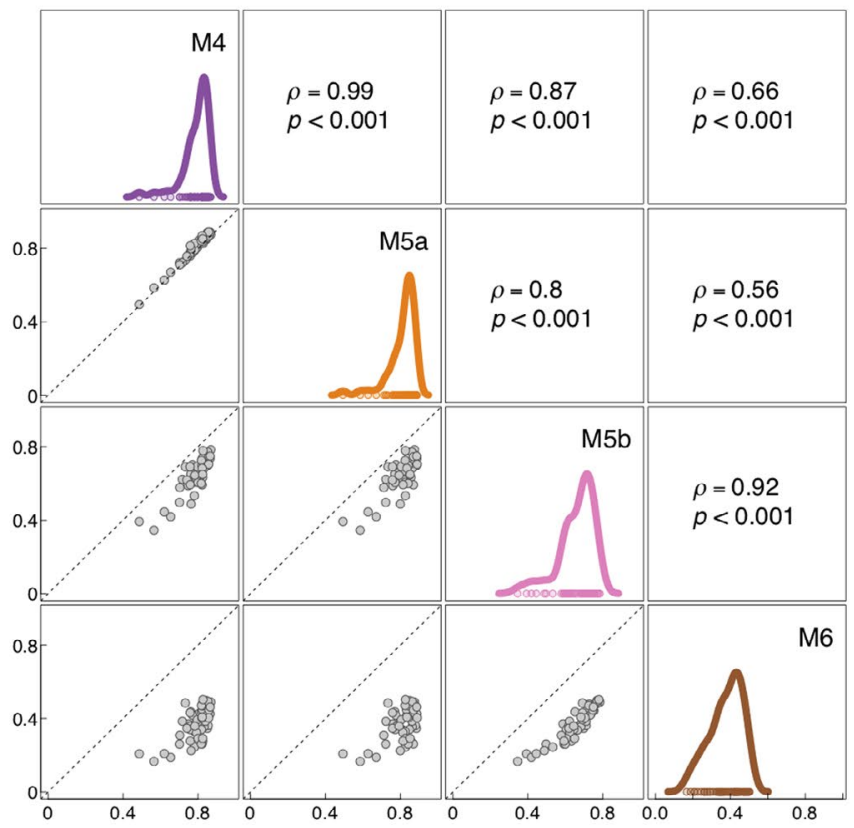

FIGURE 2 For 61 ant communities, plots show the degree of similarity, as measured by Pearson's correlation ( $\rho$ ), in values of Functional Richness (FRic) (a) and Rao (b) calculated by different methods. Each method (M1-M6) includes a different scale (or scales) of trait variability in functional diversity, based on the resolution of the trait data (see Table 1). Plots along the diagonal depict probability density functions showing the distribution of FRic or Rao values calculated by individual methods

detected by the best models of the other methods, which used trait data of lower resolution and which excluded particular scales of intraspecific trait variability. The plant model from the HighRes method (M1) detected a negative linear effect of soil water content on FRic (Figure 3a). All other methods (M2-M6) led to substantially different ecological interpretations (Figure $3 b-f: \Delta A I C c>2$ in all 

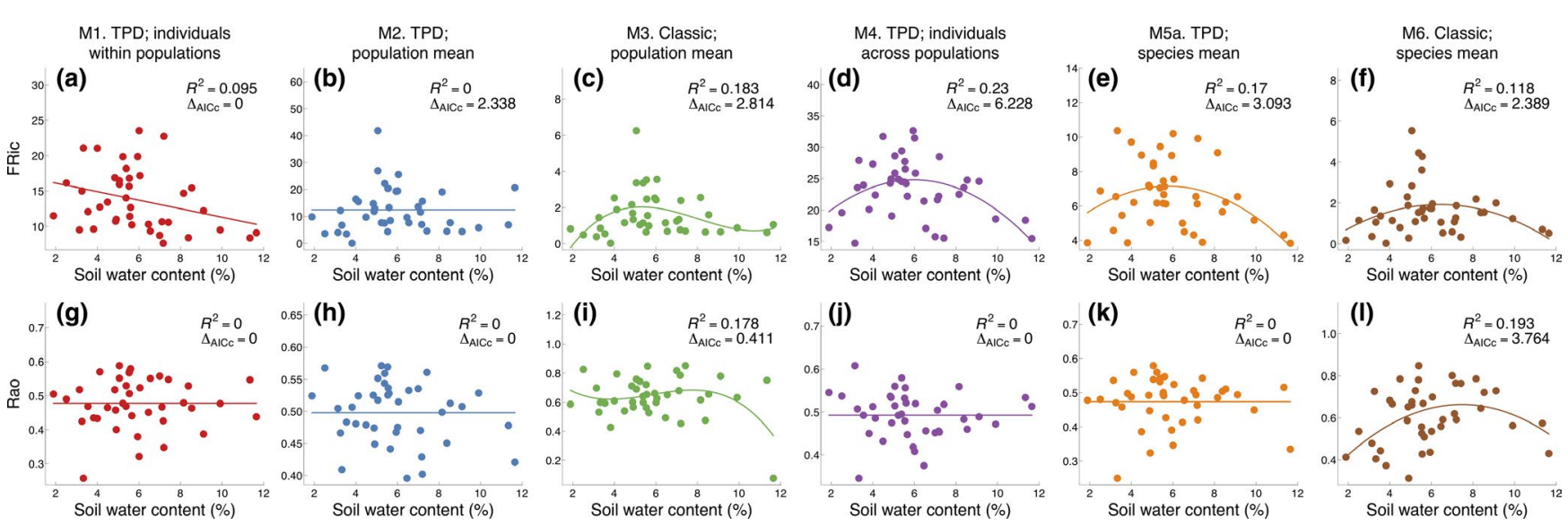

FIGURE 3 Six models of functional diversity measured in terms of the indices Functional Richness (FRic) (top) and Rao (bottom), in 40 Mediterranean plant communities (dots) distributed along a gradient of soil water content. The models were produced from six different methods (M1-M6) for calculating functional diversity, each including a different scale (or scales) of trait variability, based on the resolution of the trait data available (see Table 1). For each index, the congruence of each model from M2 to M6 with the model from M1 (which used data of the highest resolution) is summarised by an $\triangle \mathrm{AICc}$ score, where a value of 0 indicates no distortion of the ecological pattern in the $\mathrm{M} 1$ model, and increasing values indicate increasing distortion

M4. TPD; individuals across populations

(a)

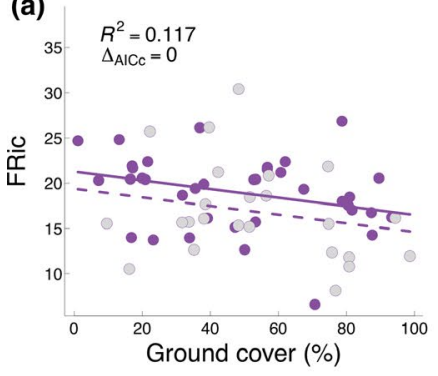

(e)

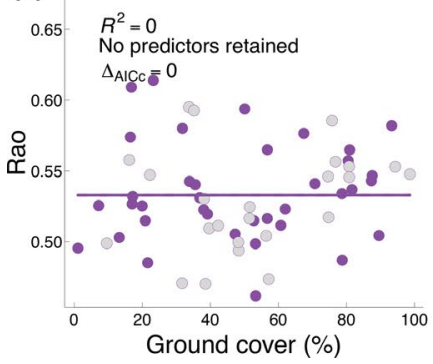

M5a. TPD; species mean (SD for each species)

(b)

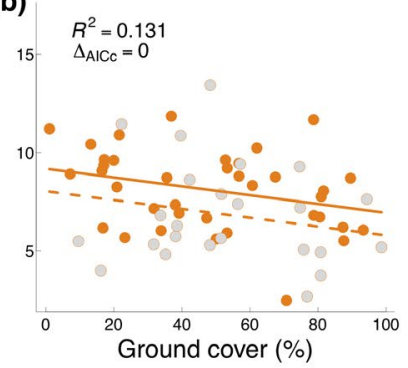

(f)

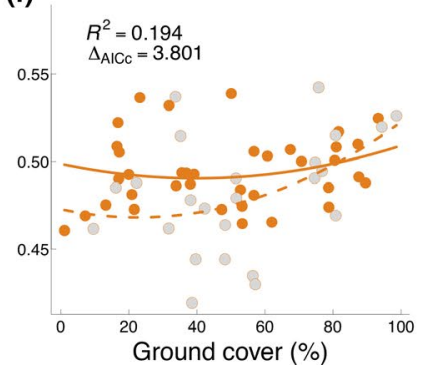

M5b. TPD; species mean (SD common across species)

(c)

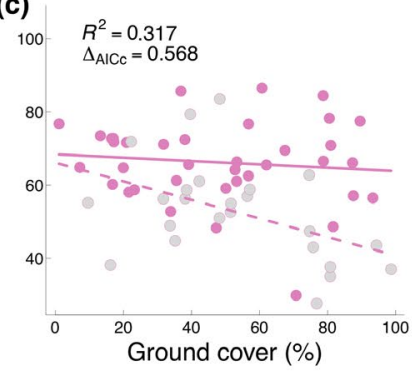

(g)

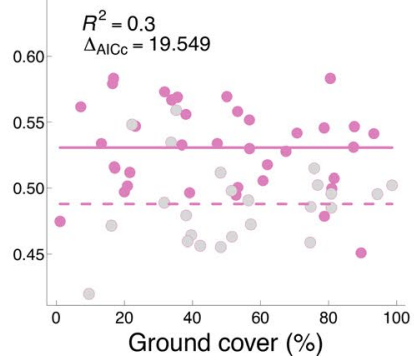

M6. Classic; species mean

(d)

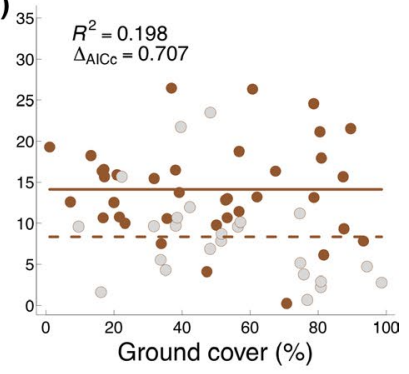

(h)

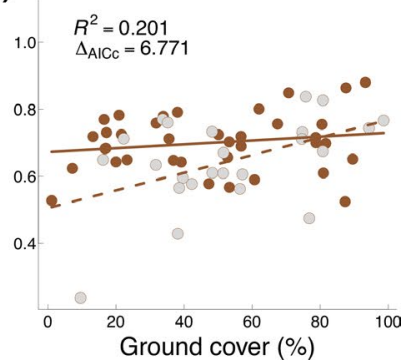

FIGURE 4 Four models of functional diversity measured in terms of the indices Functional Richness (FRic) (top) and Rao (bottom), in 61 ant communities distributed along a gradient of ground cover, including communities with (coloured dots, solid trend line) and without (grey dots, dotted trend line) an invasive species, Solenopsis invicta. The models were produced from four different methods (M4, M5a, M5b, M6) for calculating functional diversity, each including a different scale (or scales) of trait variability, based on the resolution of the trait data available (see Table 1). For each index, the congruence of the models from M5a, M5b and M6 with the model from M4 (which used data of the highest resolution) is indicated by an $\triangle \mathrm{AICC}$ score, where a value of 0 indicates no distortion of the ecological pattern in the M4 model, and increasing values indicate increasing distortion

cases). The models from M3, M4, M5a and M6 detected nonlinear changes in which FRic peaked at intermediate soil water content (Figure 3c-f) while the M2 model failed to detect an effect of soil water content on FRic (Figure 3b). The ant model from the HighRes method (M4) detected a significant negative linear effect of ground cover on FRic and a significant effect of invasion (Figure 4a). Only the M5a model detected an identical ecological pattern (Figure 4b $\triangle \mathrm{AICc}=0$ ). Though the model from $\mathrm{M} 5 \mathrm{~b}$ reproduced the significant negative linear effect of ground cover (Figure 4c), it also detected a significant interaction effect between ground cover and invasion. The model from M6 failed to detect the effect of ground cover altogether (Figure 4d). 


\section{3 | Rao responds to the exclusion of intraspecific trait variability in general}

The Rao patterns detected by the best models from HighRes methods were accurately reproduced by the best models from a few other methods which included intraspecific trait variability, but not by methods which excluded it entirely. The plant model from the HighRes method (M1) did not detect any significant effect of soil water content on Rao (Figure 3g). This pattern was accurately reproduced by models from M2, M4 and M5a (Figure 3h,j,k: $\triangle \mathrm{AICc}=0$ ). Models from both $\mathrm{M} 3$ and $\mathrm{M} 6$ detected significant quadratic relationships between Rao and soil water content (Figure 3i,I), with that from M6 leading to a substantially different ecological interpretation (Figure 3I: $\triangle \mathrm{AICC}=3.8$ ). The ant model from the HighRes method (M4) did not detect any significant effects from ground cover and invasion (Figure 4e). This pattern was not accurately reproduced by any of the other methods. The model from M5a detected a nonlinear relationship and an interaction between ground cover and invasion (Figure 4f: $\triangle \mathrm{AICc}=3.8$ ). By contrast, models from $\mathrm{M} 5 \mathrm{~b}$ and M6 detected significant effects with ground cover and invasion which led to very different ecological interpretations (Figure 4g,h: $6<\Delta \mathrm{AIC}<20$ )

\section{4 | DISCUSSION}

Our empirical findings in two ecologically disparate systems show that widely used dissimilarity-based and volume-based indices capture different functional diversity patterns, and crucially, that these indices are strongly influenced by the particular scales of trait variability included, as determined by the specific resolution of the trait data available. These results strongly suggest that basic decisions in functional diversity assessments-those about whether traits are sampled in situ, and if so, from how many and which individuals; which indices are used and how they are calculated-can largely determine the patterns observed and even alter conclusions about ecological processes entirely. As our study examined the morphological traits of plants and ants, further work is needed to explore the effects of intraspecific trait variability on the functional diversity of other taxonomic assemblages and for traits spanning organisms' physiology and behaviour. Nonetheless, in light of the findings, we suggest recommendations and issues to consider in both field-based and data-driven studies on functional diversity.

\section{1 | Use multiple indices to draw inference}

Limiting similarity (MacArthur \& Levins, 1967) has long been invoked as a powerful driver of community structure, but the extent of its influence across taxonomic groups is less explored with trait-based approaches, which have the advantage of quantifying species' niches in comparable terms (McGill et al., 2006). In our study, volume-based and dissimilarity-based functional diversity indices collectively detected patterns consistent with the effects of limiting similarity in the community structure of ecologically distinct groups such as plants and ants inhabiting different bioregions. Using trait data of the highest resolution, the best models in both groups showed that the total volume occupied by the trait values of all species (FRic) decreased along the respective environmental gradients, suggesting that the total niche space available to all members within the community was reduced (Figures 3a and 4a). However, in spite of the shrinking niche space at the community level, the overlap between species within this space-as measured by their trait dissimilarity, Rao-remained constant (Figures $3 g$ and $4 \mathrm{e}$ ). The limiting similarity hypothesis is further supported by evidence that species richness declined along the environmental gradient in both cases (Figure S2). To the best of our knowledge, this is the first study to show the importance of including intraspecific variability at the within-plot scale for detecting the effects of limiting similarity in natural assemblages. It supports previous findings in simulated (de Bello et al., 2013) and experimental conditions (Mason et al., 2011). More broadly, these results demonstrate that using different indices to target distinct facets of functional diversity can enhance inferences about ecological processes. Nonetheless, the relationships were detected using trait data of high resolution, which is not always available. Moreover, we found functional diversity indices to be very sensitive to the particular scales of trait variability included. An understanding of these relationships is therefore crucial for selecting appropriate methods and avoiding misinterpretation in functional diversity research.

\section{2 | Results from mean-based methods may distort patterns and alter inference}

In keeping with previous studies (Baraloto et al., 2010; de Bello et al., 2013), we found that calculations solely using the mean trait values of species mis-estimated functional diversity (Figure 1: values from M1 are weakly correlated with those from M6). However, our results also go further than previous work, as they empirically show that mean-based methods for calculating functional diversity in general-such as convex hull-based FRic and Gower-based Rao-can distort patterns and alter conclusions about underlying processes. For instance, a negative-linear relationship may be transformed to a quadratic one (Figure 3a vs. f); a 'false' effect of an invasive species may be detected (Type I error; Figure $4 \mathrm{e}$ vs. h) and a 'true' effect of an environmental gradient may fail to be detected (Type II error; Figure $4 a$ vs. d). Furthermore, even when an effort is made to address among-population trait variability such as by systematically sampling the traits of conspecific individuals within plots, the resultant functional diversity patterns may still be distorted if the among-population trait variability is derived solely from the mean trait values of populations (Figure 3: in both FRic and Rao, the model from M3 clearly changes the relationship modelled with M1). Given these apparent limitations of mean-based methods in preserving the integrity of functional diversity estimates and patterns, calls for using probabilistic methods to include intraspecific trait variability 
into functional diversity (Blonder et al., 2018; Carmona et al., 2016a) should not be understated. Yet, our results show that both the scales of intraspecific trait variability considered (among individuals vs. among populations) and the precise methods with which probabilistic trait distributions are estimated (e.g. M1, M2, M4, M5a, M5b) will strongly influence the functional diversity observed.

\subsection{Aim to estimate probabilistic trait distributions from trait values of individuals directly}

We recommend that ecologists sample traits of multiple individuals per species and use these values to estimate probabilistic trait distributions for each species directly with kernel density functions (e.g. in M4), even when this cannot be done for every population of every species (as in M1). In our study, such an approach, sampling at least 10 individuals of each species (M4) achieved values of FRic and Rao that were relatively similar to those calculated by the method using trait data of the highest resolution, which sampled at least 10 individuals per population of each species (M1; Figure 1).

Our results show that FRic is especially sensitive to trait variability among individuals within the same populations. We therefore suggest that assessments of FRic and community niche space can address this scale of trait variability by sampling the traits of conspecific individuals occurring within the same plots. Here, the FRic of plant communities was overestimated by methods which either estimated the trait probability density functions of species $\left(T^{2} D_{S}\right)$ from mean values or methods which used only the mean value at the population or species levels (M2, M3 and M6), as compared to methods which estimated $\mathrm{TPD}_{S}$ using the trait values of individuals directly (M4; Figure 1a: compare plots for M1 against M2, M3 and M6, with plots for M1 against M4). These relationships imply that each plant community actually occupied a smaller niche space than would be expected if it only included the among-population trait variability in each species, or if it excluded intraspecific trait variability entirely. This reduction in community niche space locally may be driven by environmental filtering or competition hierarchies promoting similarity in the traits of coexisting individuals (Carmona, de Bello, Azcárate, et al., 2019; Germain et al., 2018). Yet, the findings demonstrate that such important assembly processes may fail to be detected if among-individual trait variability is not included in functional diversity assessments.

The low correlation between the FRic values of $M 1$ and M2 (Figure 1a) was particularly surprising. Both methods capture trait variability at the same spatial and organisational scales (Table 1); the low correlation therefore arose from the different ways by which the $\mathrm{TPD}_{S}$ of populations was estimated. Estimating TPDS directly using kernel density functions and all sampled values (M1) and estimating them as multivariate normal distributions centred in the mean sampled values (M2) produced distinct results. This suggests that FRic is strongly influenced by the effects of individuals with extreme trait values (in spite of TPD methods theoretically being more robust to such effects than convex hull-based hypervolumes; Carmona et al., 2016a). This sensitivity of FRic to extreme values could be investigated further by testing the effects of setting smaller probability thresholds to the TPD (see Blonder, 2016; Carmona et al. 2016b).

We found that Rao, like FRic, was sensitive to the exclusion of intraspecific trait variability overall (Figures 1-4: focusing on Rao, compare M6 to M1 or M4 for plants and ants, respectively). Yet, in contrast to the patterns observed for FRic, the methods estimating species' trait probability density functions directly from individual values using kernel density functions (M1, M4) and those estimating them as multivariate normal distributions centred in the mean trait value (M2, M5a) produced similar levels of Rao (Figure 1b: M1 vs. M2 for plants; Figure 2b: M4 vs. M5a for ants). Furthermore, Rao calculated by these different methods followed similar trends with environmental gradients (Figure 3g,h,j,k; but see Figure 4e,f despite the very high correlation between M4 and M5a for Rao in the ant data). These results are encouraging-although distinct, the two methods are evidently valid alternatives for calculating trait dissimilarities between populations or species. Importantly, the results also suggest that estimations of dissimilarities between species (e.g. with Rao) are more robust to methodological choices than estimations of the total functional space occupied by communities (e.g. FRic).

Observed functional diversity will most likely approximate reality when trait probability density functions are estimated directly using (a) the trait values of multiple individuals per species and (b) information on the spatial structure of their populations across environments (Carmona et al., 2016a). Yet, large samples of trait measurements and spatially detailed trait information are seldom available. With respect to these limitations of empirical studies, our results suggest that even with a lower resolution in trait data, intraspecific trait variability can still be included in dissimilarity-based functional diversity without leading to significant distortion in ecological patterns. This is in agreement with previous results suggesting that trait sampling is more efficient when local (i.e. measured in the corresponding plot) trait values are used, rather than a regional average for each species (Baraloto et al., 2010; Carmona et al., 2015; Gross et al., 2013). In the specific case of the TPD framework, this can be achieved using variance estimators to estimate the $\mathrm{TPD}_{S}$ of each species as a multivariate normal trait distribution centred in the sampled mean trait value (e.g. M2, M5a).

In estimating the trait distributions of species with variance estimators, however, one should not assume that the same algorithm is valid for estimating the variance in the distributions of trait values of disparate taxa. This is evidenced by our finding that the FRic of ant communities from when $\mathrm{TPD}_{S}$ was estimated assuming a constant variance (M5b; following the solution proposed in a plant-based study; Lamanna et al., 2014) was over two times higher than their FRic from when $\mathrm{TPD}_{S}$ was estimated using the trait values of all sampled individuals (M4), as well as that from when $\mathrm{TPD}_{S}$ was estimated using the variance in the data (M5a; Figure 4a-c). Specifically, this suggests that in comparison to plants, trait variation within ant species may be very low relative to trait variation between species (as reported in Gaudard et al., 2019). In any case, these results strongly suggest that decisions about the precise variances used for 
estimating trait distributions should be grounded in the ecological characteristics of the studied organisms. Caution is therefore advised for multi-taxa analyses, where it may seem temptingly efficient to apply the same estimation procedure across all groups. Further studies on the contribution of intraspecific variability to total trait variability in different taxa (e.g. Siefert et al., 2015) are needed for navigating these issues.

\section{5 | CONCLUSIONS}

This study demonstrates the strong influence that trait variability within species can have on our view of functional diversity and underlying ecological processes. It also clarifies when and how intraspecific trait variability can be reasonably included in functional diversity studies with limited trait data. Accounting for this crucial yet overlooked source of functional variability in nature will be key to understanding the responses and effects of biodiversity.

\section{ACKNOWLEDGEMENTS}

M.K.L.W. is supported by a grant from the National Geographic Society (60-16) and a Clarendon Scholarship from the University of Oxford. C.P.C. was supported by the Estonian Research Council (project PSG293) and the European Union through the European Regional Development Fund (Centre of Excellence EcolChange). The authors thank Owen Lewis, Benoit Guénard, Michelle Jackson, Catherine Parr as well as Stefano Mammola and an anonymous reviewer for comments on previous versions of the manuscript.

\section{AUTHORS' CONTRIBUTIONS}

Both authors designed the research, collected the data from the field, performed the trait measurements and data analyses. M.K.L.W. led the writing of the manuscript with substantial contributions from C.P.C.

\section{DATA AVAILABILITY STATEMENT}

Data and code are deposited in the Dryad Digital Repository https:// doi.org/10.5061/dryad.612jm642j (Carmona \& Wong, 2020).

\section{ORCID}

Mark K. L. Wong (iD https://orcid.org/0000-0002-6248-3103

Carlos P. Carmona iD https://orcid.org/0000-0001-6935-4913

\section{REFERENCES}

Abràmoff, M. D., Magalhães, P. J., \& Ram, S. J. (2004). Image processing with Image J. Biophotonics International, 11, 36-42.

Albert, C. H., Grassein, F., Schurr, F. M., Vieilledent, G., \& Violle, C. (2011). When and how should intraspecific variability be considered in trait-based plant ecology? Perspectives in Plant Ecology, Evolution and Systematics, 13, 217-225. https://doi.org/10.1016/j. ppees.2011.04.003

Ames, G. M., Anderson, S. M., \& Wright, J. P. (2016). Multiple environmental drivers structure plant traits at the community level in a pyrogenic ecosystem. Functional Ecology, 30, 789-798. https://doi. org/10.1111/1365-2435.12536

Baraloto, C., Timothy Paine, C. E., Patino, S., Bonal, D., Hérault, B., \& Chave, J. (2010). Functional trait variation and sampling strategies in species-rich plant communities. Functional Ecology, 24, 208-216. https://doi.org/10.1111/j.1365-2435.2009.01600.x

Barton, K. (2016). MuMIn: Multi-model inference. R package version 1.6.

Baselga, A., Orme, D., Villeger, S., De Bortoli, J., Leprieur, F., \& Baselga, M. A. (2018). Package 'betapart'. Partitioning beta diversity into turnover and nestedness components, version, $1(0)$.

Blonder, B. (2016). Pushing past boundaries for trait hypervolumes: A response to Carmona et al. Trends in Ecology \& Evolution, 31, 665-667. https://doi.org/10.1016/j.tree.2016.07.001

Blonder, B., Morrow, C. B., Maitner, B., Harris, D. J., Lamanna, C., Violle, C., Enquist, B. J., \& Kerkhoff, A. J. (2018). New approaches for delineating $\mathrm{n}$-dimensional hypervolumes. Methods in Ecology and Evolution, 9, 305-319.

Botta-Dukát, Z. (2005). Rao's quadratic entropy as a measure of functional diversity based on multiple traits. Journal of Vegetation Science, 16, 533-540. https://doi.org/10.1111/j.1654-1103.2005.tb02393.x

Burnham, K. P., \& Anderson, D. R. (2002). Model selection and inference: A practical information-theoretic approach (2nd ed., pp. 488). Springer-Verlag.

Carmona, C. P., de Bello, F., Azcárate, F. M., Mason, N. W., \& Peco, B. (2019). Trait hierarchies and intraspecific variability drive competitive interactions in Mediterranean annual plants. Journal of Ecology, 107, 2078-2089.

Carmona, C. P., de Bello, F., Mason, N. W., \& Lepš, J. (2016). Traits without borders: Integrating functional diversity across scales. Trends in Ecology \& Evolution, 31, 382-394.

Carmona, C. P., de Bello, F., Mason, N. W., \& Lepš, J. (2016). The density awakens: A reply to Blonder. Trends in Ecology \& Evolution, 31, 667-669.

Carmona, C. P., de Bello, F., Mason, N. W., \& Lepš, J. (2019). Trait probability density (TPD): Measuring functional diversity across scales based on TPD with R. Ecology, 100, e02876.

Carmona, C. P., Rota, C., Azcárate, F. M., \& Peco, B. (2015). More for less: Sampling strategies of plant functional traits across local environmental gradients. Functional Ecology, 29, 579-588.

Carmona, C. P., \& Wong, M. K. L. (2020). Data from: Including intraspecific trait variability to avoid distortion of functional diversity and ecological inference: Lessons from natural assemblages. Dryad Digital Repository, https://doi.org/10.5061/dryad.612jm642j

Cianciaruso, M. V., Batalha, M. A., Gaston, K. J., \& Petchey, O. L. (2009). Including intraspecific variability in functional diversity. Ecology, 90, 81-89.

Cornwell, W. K., Schwilk, D. W., \& Ackerly, D. D. (2006). A trait-based test for habitat filtering: Convex hull volume. Ecology, 87, 1465-1471.

de Bello, F., Carmona, C. P., Lepš, J., Szava-Kovats, R., \& Pärtel, M. (2016). Functional diversity through the mean trait dissimilarity: Resolving shortcomings with existing paradigms and algorithms. Oecologia, 180, 933-940.

de Bello, F., Carmona, C. P., Mason, N. W. H., Sebastià, M.-T., \& Lepš, J. (2013). Which trait dissimilarity for functional diversity: Trait means or trait overlap? Journal of Vegetation Science, 24, 807-819.

Des Roches, S., Post, D. M., Turley, N. E., Bailey, J. K., Hendry, A. P., Kinnison, M. T., Schweitzer, J. A., \& Palkovacs, E. P. (2018). The ecological importance of intraspecific variation. Nature Ecology and Evolution, 2, 57-64. https://doi.org/10.1038/s41559-017-0402-5

Díaz, S., Kattge, J., Cornelissen, J. H. C., Wright, I. J., Lavorel, S., Dray, S., Reu, B., Kleyer, M., Wirth, C., Colin Prentice, I., Garnier, E., Bönisch, G., Westoby, M., Poorter, H., Reich, P. B., Moles, A. T., Dickie, J., Gillison, A. N., Zanne, A. E., ... Gorné, L. D. (2016). The global spectrum of plant form and function. Nature, 529, 167-171. https://doi. org/10.1038/nature16489 
Duong, T. (2015). ks: Kernel density estimation and kernel discriminant analysis for multivariate data in R. Journal of Statistical Software, 21(7), 1-16.

Gaudard, C. A., Robertson, M. P., \& Bishop, T. R. (2019). Low levels of intraspecific trait variation in a keystone invertebrate group. Oecologia, 190, 725-735. https://doi.org/10.1007/s00442-019-04426-9

Germain, R. M., Mayfield, M. M., \& Gilbert, B. (2018). The 'filtering' metaphor revisited: Competition and environment jointly structure invasibility and coexistence. Biology Letters, 14, 20180460. https://doi. org/10.1098/rsbl.2018.0460

Gotelli, N. J., \& Arnett, A. E. (2000). Biogeographic effects of red fire ant invasion. Ecology Letters, 3, 257-261. https://doi.org/10.1046/ j.1461-0248.2000.00138.x

Gross, N., Börger, L., Soriano-Morales, S. I., Le Bagousse-Pinguet, Y., Quero, J. L., García-Gómez, M., Valencia-Gómez, E., \& Maestre, F. T. (2013). Uncovering multiscale effects of aridity and biotic interactions on the functional structure of Mediterranean shrublands. Journal of Ecology, 101, 637-649. https://doi.org/10.1111/1365-2745.12063

Gross, N., Le Bagousse-Pinguet, Y., Liancourt, P., Berdugo, M., Gotelli, N. J., \& Maestre, F. T. (2017). Functional trait diversity maximizes ecosystem multifunctionality. Nature Ecology and Evolution, 1, 1-9. https://doi.org/10.1038/s41559-017-0132

Laliberté, E., \& Legendre, P. (2010). A distance-based framework for measuring functional diversity from multiple traits. Ecology, 91, 299305. https://doi.org/10.1890/08-2244.1

Laliberté, E., Legendre, P., Shipley, B., \& Laliberté, M. E. (2014). Package 'FD'. Measuring functional diversity from multiple traits, and other tools for functional ecology.

Lamanna, C., Blonder, B., Violle, C., Kraft, N. J. B., Sandel, B., imova, I., Donoghue, J. C., Svenning, J.-C., McGill, B. J., Boyle, B., Buzzard, V., Dolins, S., Jorgensen, P. M., Marcuse-Kubitza, A., Morueta-Holme, N., Peet, R. K., Piel, W. H., Regetz, J., Schildhauer, M., ... Enquist, B. J. (2014). Functional trait space and the latitudinal diversity gradient. Proceedings of the National Academy of Sciences of the United States of America, 111, 13745-13750. https://doi.org/10.1073/pnas.13177 22111

MacArthur, R., \& Levins, R. (1967). The limiting similarity, convergence, and divergence of coexisting species. The American Naturalist, 101, 377-385. https://doi.org/10.1086/282505

Mammola, S., \& Cardoso, P. (2020). Functional diversity metrics using kernel density n-dimensional hypervolumes. Methods in Ecology and Evolution, 11, 986-995.

Martello, F., De Bello, F., de Castro Morini, M. S., Silva, R. R., de Souza-Campana, D. R., Ribeiro, M. C., \& Carmona, C. P. (2018). Homogenization and impoverishment of taxonomic and functional diversity of ants in Eucalyptus plantations. Scientific Reports, 8, 3266. https://doi.org/10.1038/s41598-018-20823-1

Mason, N. W. H., de Bello, F., Doležal, J., \& Lepš, J. (2011). Niche overlap reveals the effects of competition, disturbance and contrasting assembly processes in experimental grassland communities. Journal of Ecology, 99, 788-796. https://doi.org/10.1111/j.1365-2745.2011.01801.x

McGill, B. J., Enquist, B. J., Weiher, E., \& Westoby, M. (2006). Rebuilding community ecology from functional traits. Trends in Ecology \& Evolution, 21, 178-185. https://doi.org/10.1016/j.tree.2006.02.002

Messier, J., McGill, B. J., \& Lechowicz, M. J. (2010). How do traits vary across ecological scales? A case for trait-based ecology. Ecology Letters, 13, 838-848. https://doi.org/10.1111/j.1461-0248.2010.01476.x

Mouchet, M. A., Villéger, S., Mason, N. W., \& Mouillot, D. (2010). Functional diversity measures: An overview of their redundancy and their ability to discriminate community assembly rules. Functional Ecology, 24, 867-876. https://doi.org/10.1111/j.1365-2435.2010.01695.x

Petchey, O. L., \& Gaston, K. J. (2002). Functional diversity (FD), species richness and community composition. Ecology Letters, 5, 402-411. https://doi.org/10.1046/j.1461-0248.2002.00339.x

Pigot, A. L., Sheard, C., Miller, E. T., Bregman, T. P., Freeman, B. G., Roll, U., Seddon, N., Trisos, C. H., Weeks, B. C., \& Tobias, J. A. (2020). Macroevolutionary convergence connects morphological form to ecological function in birds. Nature Ecology and Evolution, 4, 230-239. https://doi.org/10.1038/s41559-019-1070-4

R Core Team. (2017). R: A language and environment for statistical computing.

Siefert, A., Violle, C., Chalmandrier, L., Albert, C. H., Taudiere, A., Fajardo, A., Aarssen, L. W., Baraloto, C., Carlucci, M. B., Cianciaruso, M. V., de L. Dantas, V., de Bello, F., Duarte, L. D. S., Fonseca, C. R., Freschet, G. T., Gaucherand, S., Gross, N., Hikosaka, K., Jackson, B., ... Wardle, D. A. (2015). A global meta-analysis of the relative extent of intraspecific trait variation in plant communities. Ecology Letters, 18, 14061419. https://doi.org/10.1111/ele.12508

Traba, J., Iranzo, E. C., Carmona, C. P., \& Malo, J. E. (2017). Realised niche changes in a native herbivore assemblage associated with the presence of livestock. Oikos, 126, 1400-1409. https://doi.org/10.1111/ oik.04066

van der Plas, F., Van Klink, R., Manning, P., Olff, H., \& Fischer, M. (2017). Sensitivity of functional diversity metrics to sampling intensity. Methods in Ecology and Evolution, 8, 1072-1080. https://doi. org/10.1111/2041-210X.12728

Villéger, S., Mason, N. W., \& Mouillot, D. (2008). New multidimensional functional diversity indices for a multifaceted framework in functional ecology. Ecology, 89, 2290-2301. https://doi.org/10.1890/07-1206.1

Weiher, E., Clarke, G. P., \& Keddy, P. A. (1998). Community assembly rules, morphological dispersion, and the coexistence of plant species. Oikos, 81, 309-322. https://doi.org/10.2307/3547051

Weiss, K. C., \& Ray, C. A. (2020). Unifying functional trait approaches to understand the assemblage of ecological communities: Synthesizing taxonomic divides. Ecography, 42, 2012-2020. https://doi.org/ 10.1111/ecog.04387

Westneat, D. F., Wright, J., \& Dingemanse, N. J. (2015). The biology hidden inside residual within-individual phenotypic variation. Biological Reviews, 90, 729-743. https://doi.org/10.1111/brv.12131

Wong, M. K. L., Guénard, B., \& Lewis, O. T. (2020). The cryptic impacts of invasion: Functional homogenization of tropical ant communities by invasive fire ants. Oikos, 129, 585-597. https://doi.org/10.1111/ oik.06870

\section{SUPPORTING INFORMATION}

Additional supporting information may be found online in the Supporting Information section.

How to cite this article: Wong MKL, Carmona CP. Including intraspecific trait variability to avoid distortion of functional diversity and ecological inference: Lessons from natural assemblages. Methods Ecol Evol. 2021;00:1-12. https://doi. org/10.1111/2041-210X.13568 